\title{
Improved reproducing kernel method to solve space-time fractional advection-dispersion equation
}

\author{
Tofigh Allahviranloo ${ }^{1}$, Hussein Sahihi ${ }^{1}$, Soheil Salahshour ${ }^{2}$, and D. Baleanu ${ }^{3}$ \\ ${ }^{1}$ Bahcesehir Universitesi \\ ${ }^{2}$ Bahcesehir University \\ ${ }^{3}$ Canakkale Onsekiz Mart Universitesi
}

January 1, 2021

\begin{abstract}
In this paper, we consider the Space-Time Fractional Advection-Dispersion equation on a finite domain with variable coefficients. Fractional Advection- Dispersion equation as a model for transporting heterogeneous subsurface media as one approach to the modeling of the generally non-Fickian behavior of transport. We use a semi-analytical method as Reproducing kernel Method to solve the Space-Time Fractional Advection-Dispersion equation so that we can get better approximate solutions than the methods with which this problem has been solved. The main obstacle to solve this problem is the existence of a Gram-Schmidt orthogonalization process in the general form of the reproducing kernel method, which is very time-consuming. So, we introduce the Improved Reproducing Kernel Method, which is a different implementation for the general form of the reproducing kernel method. In this method, the Gram-Schmidt orthogonalization process is eliminated to significantly reduce the CPU-time. Also, the present method increases the accuracy of approximate solutions.
\end{abstract}

\section{Hosted file}

Paper 8s.pdf available at https://authorea.com/users/346196/articles/502043-improvedreproducing-kernel-method-to-solve-space-time-fractional-advection-dispersion-equation 
figures/aa/aa-eps-converted-to.pdf 
figures/ddE/ddE-eps-converted-to.pdf 
figures/ddE2/ddE2-eps-converted-to.pdf 
figures/dE/dE-eps-converted-to.pdf 
figures/dE2/dE2-eps-converted-to.pdf 
figures/E/E-eps-converted-to.pdf 